\title{
What Lies Behind the Diagnosis of Acute Pneumonia?
}

Igor Klepikov*

Professor, USA

*Corresponding author: Igor Klepikov, Professor, USA

Submission: 眥 November 13, 2018; Published: 㘹 November 20, 2018

\section{Open Letter to the Editor}

The reason for my present appeal is to draw the attention of a wide range of experts to long-known and proven scientific facts, which, despite their importance, remain unclaimed. Specialists in the field of inflammatory diseases of the lungs are undoubtedly familiar with modern views on the nature of acute pneumonia (AP), the principles of their treatment and the tendency to reduce the effectiveness of this aid. Therefore, I hope that the problem, the essence of which I want to draw attention, will be understood by readers without a detailed review of the literature. When I was forced to do research in this area 40 years ago, the overall situation in the world looked more prosperous. Therefore, even despite the impressive results, my voice was not heard [1]. To date, it has become obvious to many that neither the constant updating of antimicrobials and the production of more effective drugs, nor the long-term "vaccination against pneumonia" not only does not reduce the number of complicated forms of the disease but does not even hinder their growth. And the main reason for this situation is the false idea of the essence of the disease as an exclusively infectious process. AP has been known to medicine for thousands of years and unlike many others, infection has never been and is not now regarded as a form of disease that requires isolation of the patient and compliance with special epidemiological requirements. The only exception may be patients with a clear viral etiology onset of the disease during periods of widespread epidemics.

The causative agents of AP in the bulk of patients are representatives of symbiotic microflora, which may include today appeared in recent years antibiotic resistant strains. It is also necessary to recall the well-known fact that the carrier of even very virulent microbes does not mean the mandatory development of the disease. In other words, microflora is only an example of the etiological factors of AP and additional conditions are needed to start it. Today, few people remember the evidence of witnesses of the first clinical trials of antibiotics (if at all heard about them), when literally two or three injections eliminated the signs of AP. Almost instantaneous elimination of one of the most important causes of the disease allowed to interrupt the further development of the inflammatory process, which helped the body to eliminate existing structural changes of tissues without problems. The effectiveness of antibiotic therapy at the present stage is very far from the assessment of its initial application and this is quite understandable. Microbes, as living biological objects, can adapt to external influences and for a long period of antibiotic use they have achieved a certain resistance to these drugs. In the pre-antibiotic era Streptococcus pneumoniae was invariably the leading causative agent of AP. After the introduction of antibiotics into clinical practice, the change of leaders among pathogens of AP has become commonplace.

Unfortunately, in the new conditions of the consequences of antibiotic therapy, classical medicine changes only therapeutic tactics, leaving unchanged the strategy of solving the problem. Belief in the limitless possibilities of antibiotics continues to dominate the definition of strategic approaches to the treatment of AP. At the same time, it is well known that the development and production of antibiotics of a new generation is carried out in connection with the appearance of non-standard strains. That is, tactical changes are the result of the emergence of new conditions, not an attempt to outrun them. The absence of reasoned explanations for the continued increase in the number of complicated forms of AP, the emergence and growth of the so-called sterile empyema, the development of purulent complications of the disease contrary to the use of the most effective antibiotics indicate stagnation in the scientific assessment of the nature and essence of AP. The reason for this stagnation lies on the surface, because more than one generation of doctors has been educated and trained based on the exceptional role and reverence before antibiotics. In recent scientific publications on this topic, it is increasingly possible to meet the interpretation of AP as an infectious disease. If we focus on the etiology of the disease, it would be more logical to consider AP as an auto-infectious process, rather than as a result of receiving an infection from outside. However, the most striking and inexplicable fact is the complete disregard and neglect of classical generally recognized and undoubted scientific materials. As far as everyone knows, AP is an inflammatory process, isn't it? And if it is really an inflammation, then why do not consider the cardinal and sufficiently studied mechanisms of its development? 
Firstly, the main structural changes in the inflamed tissue are due to vascular reaction, which has a clear sequence. The dramatic result of this reaction is a significant increase in vascular permeability with the development of edema and tissue infiltration. By the way, the sequence of classical pathoanatomical stages of severe pneumonia (including the stages of red and gray hepatization) has long been described and presented in the scientific and educational literature. Secondly, acute non-specific inflammation, regardless of the pathogen, is accompanied by 5 classic signs, which were described by Celsius and Galen and are known to medicine for many centuries. The most important is the fifth sign (violation of function), which determines the specificity and peculiarities of clinical manifestations of the disease. Third, among all known nonspecific inflammatory diseases, AP is the only process that occurs in the pool of the small circle of blood circulation. It is known and proved that the circulation in the periphery is completely dependent on the circulation of the small circle of blood circulation (as well as Vice versa). In addition. there is a high reflexogenic sensitivity of the small circle vessels, which can respond to the slightest irritation, including changes in the chemical and electrolyte composition of tissues. This list of important features of the development of the inflammatory process and their effect on various lung functions can be continued. However, even the above reminders make it possible to note the importance of ideas about the nature of AP for the choice and justification of medical care.

If we continue to consider AP as an infection, it is logical to direct all efforts primarily to its suppression. In this version we return to the current situation. The results of this strategy are known, but the problem remains relevant for many years. If we consider AP as an auto-infectious inflammation in the zone of the small circle of blood circulation, it significantly changes the understanding of cause-effect relationships, the leading mechanisms of the disease and the priorities of medical care. It is a new view on the basis and essence of the AP allowed the author of these lines to justify and test pathogenetic approaches of medical care and get impressive results. In the end, the results of previous studies and clinical trials were translated into English and published in a separate publication, which is available to any interested person [2].

\section{References}

1. Klepikov I (1989) Acute pneumonia and its purulent and destructive complications in children in the midst of a major industrial centre of Western Siberia. Dissertation for the degree of Doctor of Medical Science, Siberia, Russia.

2. Klepikov I (2017) Acute pneumonia: A new look at the old problem. Lambert Academic Publishing, Latvia, ISBN: 978-3-330-35250-6.

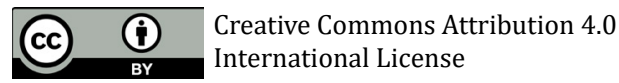

For possible submissions Click Here

\section{Submit Article}

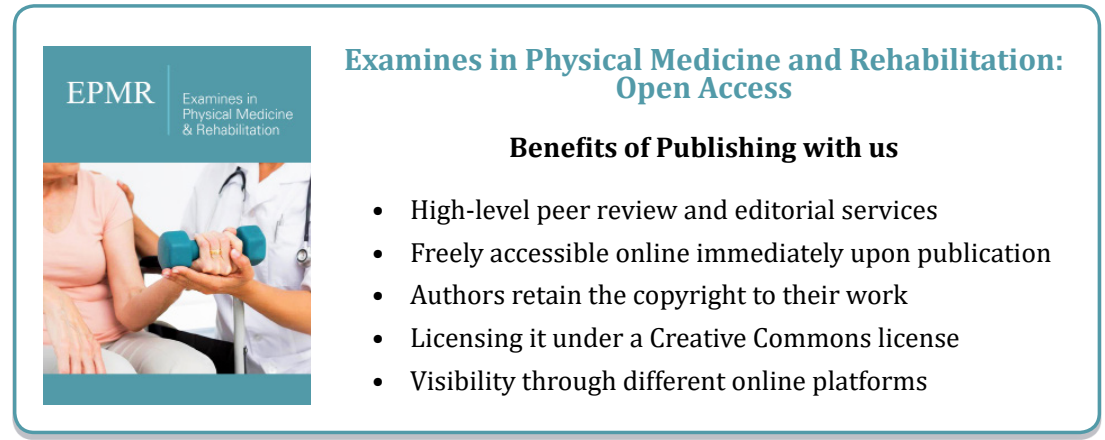

